\title{
Synthesis of new isoxazole derivatives with larvicidal activity
}

\author{
Diana C. B. da Silva-Alves, Janaína V. dos Anjos, Nery N. M. Cavalcante, Daniela M. \\ do A. F. Navarro, Geanne K. N. Santos and Rajendra M. Srivastava* \\ Departamento de Química Fundamental, Universidade Federal de Pernambuco, Cidade Universitária, \\ 50740-540, Recife, PE, Brazil
}

*Corresponding author: rms@ufpe.br

Keywords: Aedes aegypti, click chemistry, isoxazoles.

\section{INTRODUCTION}

One of the major routes of detoxification (removal of free radicals) in Aedes aegypti mosquitos is the kynurenine pathway. In this biochemical pathway, the most important reaction is the conversion of 3-hydroxykynurenine (3-HK), a harmful metabolite that leads to insect death, into xanthurenic acid, a nontoxic substance. This conversion is performed by the enzyme 3-hydroxy-kynurenine transaminase (HKT). ${ }^{1}$ As a part of drug discovery program that has the main goal of finding new larvicide candidates, we have found that some heterocycles containing both aryl and propionic acid moieties may act as ligands for HKT, having larvicidal activity against $A$. aegypti species, the dengue vector. In this work, we describe the synthesis and the larvicidal evaluation tests for new isoxazole derivatives thar might becapable of accumulate 3-HK in insects, by inhibiting the enzyme HKT detoxification reaction.

\section{RESULTS AND DISCUSSION}

To synthesize the desired compounds via coppercatalyzed cycloaddition, we started with the imidoyl chlorides (3a-j) preparation. These compounds are transformed into the real dipoles, the nitrile oxides, in situ, by means of an inorganic base such as potassium bicarbonate. The imidoyl chlorides are obtained from the corresponding aryloximes $(2 \mathbf{a}-\mathbf{j})$ and $\mathrm{N}$ chlorosuccinimide (NCS), using DMF as solvent. For the synthesis of 3-(3-aryl-isoxazol-5-yl)-propan-1-ols (4a-j), 3a-j reacted with 4-pentyn-1-ol, using $\mathrm{Cu}(\mathrm{OAc})_{2}$ and sodium ascorbate as catalytic system in the presence of potassium bicarbonate. ${ }^{2}$ In a similar way, 3-(3-aryl-isoxazol-5-yl)-propionic acids (5a-g) were prepared in the reaction between $3 \mathbf{a}-\mathbf{j}$ and sodium 4pentynoate using the same reaction conditions. Finally, we submitted two acids (5a-b) to classical esterification conditions in methanol, obtaining $\mathbf{6 a - b}$ as products (Figure). This modification was performed to verify what moiety is most important to maintain larvicidal activity: acid, alcohol or methyl ester. The larvicidal tests were performed according to procedures already reported in the literature. ${ }^{3}$ For these tests, we used $A$. aegypti larvae in the $\mathrm{L} 4$ growth stage. The results are summarized in the table and are expressed by means of $\mathrm{LC}_{50}$ (lethal dose; dose which causes the death of $50 \%$ of the group test).

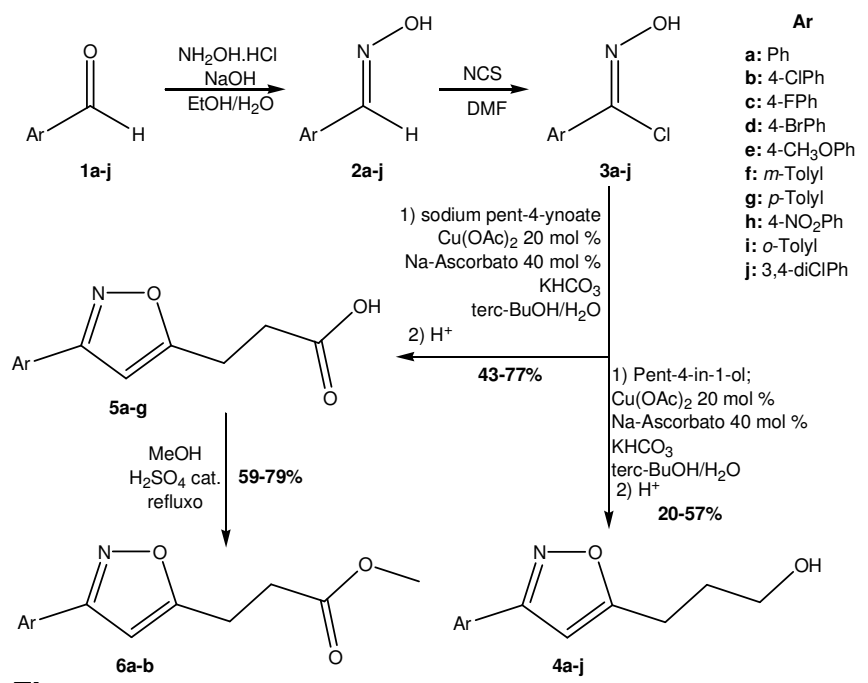

Figure.

Table. Isoxazole derivatives synthesized and their $\mathrm{LC}_{50}$ values

\begin{tabular}{ccc|ccc}
\hline Comp. & Yield & LC $_{50}(\mathbf{p p m})$ & Comp. & Yield & LC $_{50}(\mathbf{p p m})$ \\
\hline $\mathbf{4 a}$ & $54 \%$ & $>100$ & $\mathbf{5 a}$ & $63 \%$ & 82.8 \\
$\mathbf{4 b}$ & $20 \%$ & 28.9 & $\mathbf{5 b}$ & $50 \%$ & 37.7 \\
$\mathbf{4 c}$ & $33 \%$ & $>100$ & $\mathbf{5 c}$ & $77 \%$ & 33.7 \\
$\mathbf{4 d}$ & $57 \%$ & 33.3 & $\mathbf{5 d}$ & $45 \%$ & 13.8 \\
$\mathbf{4 e}$ & $20 \%$ & $>100$ & $\mathbf{5 e}$ & $43 \%$ & 35.7 \\
$\mathbf{4 f}$ & $57 \%$ & $>100$ & $\mathbf{5 f}$ & $52 \%$ & $>100$ \\
$\mathbf{4 g}$ & $52 \%$ & $>100$ & $\mathbf{5 g}$ & $48 \%$ & 38.9 \\
$\mathbf{4 h}$ & $44 \%$ & 82.0 & $\mathbf{6 a}$ & $59 \%$ & 13.2 \\
$\mathbf{4 i}$ & $22 \%$ & $>100$ & $\mathbf{6 b}$ & $79 \%$ & 3.4 \\
$\mathbf{4 j}$ & $\mathbf{4} \%$ & 17.1 & & & \\
\hline
\end{tabular}

\section{CONCLUSION}

We have accomplished the synthesis and the larvicidal evaluation tests for nineteen new isoxazole derivatives. These substances were even more potent against $A$. aegypti larvae than the ones earlier synthesized by our research group. ${ }^{4}$

\section{ACKNOWLEDGEMENTS}

CNPq, PRONEX-FACEPE.

\section{REFERENCES}

${ }^{1} \mathrm{Han}$, Q. et al. J. Biol. Chem. 2006, 281, 37175.

${ }^{2}$ Himo, F. et al. J. Am. Chem. Soc. 2005, 127, 210.

${ }^{3}$ Navarro, D.M.A.F. et al. J. Appl. Ent. 2003, 127, 46.

${ }^{4}$ Neves Filho, R. A. W. et al. Chem. Pharm. Bull. 2009, 57, 819. 